\title{
Research on Fault Diagnosis for Rotor System based on Improved LMD and Singular Value Decomposition
}

\author{
Hong Zhang \\ Weifang University, Weifang 261061, China \\ zhanghongwf@126.com
}

\begin{abstract}
Based on the disadvantages of the original LMD algorithm in terms of smoothing method and end effect, this paper puts forward a fault diagnosis method of rotor system based on the improved LMD and singular value decomposition. The PF (Product function) component obtained after applying the improved LMD method is able to separate the aliasing modes while maintaining the natures and features of the original PF component. Thus, the system's real information can be grasped more effectively. According to the experimental analysis, this method can be applied to fault diagnosis of rotor system effectively.
\end{abstract}

Keywords: Power machinery, Fault diagnosis, LMD method, Singular value decomposition

\section{Introduction}

It is unrealistic that power mechanical equipments are running without failure. To carry out research on mechanical fault diagnosis technology is of important practical significance and can greatly improve the competitiveness and sustainable development capacity of industrial enterprises. The rotor is one of the core components of power machinery. The rotor system fault diagnosis is essential in machinery and equipment manufacturing, installation and maintenance.

This paper mainly takes rotor system fault diagnosis for example. It analyzes the structural characteristics and dynamics features of the rotor system. Based on the nonstationary characteristics of the vibration signals of the rotor system faults, it proposes a method of rotor system fault diagnosis on a basis of the improved LMD (Local mean decomposition) and singular value decomposition [1]. Experimental results show that this method can be effectively applied to the rotor system fault diagnosis.

\section{The Rotor System Fault and Its Diagnosis}

Generally, the rotor system fault is the result of the coupling of a variety of faults. A machine often fails accompanied by abnormal vibrations and noises. This is an important characteristic. The vibration signals often reflect the fault information. Imbalance, misalignment, rubbing, oil whirl, etc. are the rotor system faults commonly.

\subsection{Common Rotor System Fault Types}

2.1.1. Rotor Imbalance: The rotor of the power machinery is often affected by processing, assembly errors and etc. Thus, the center of mass and the center of shape are eccentric. If the eccentricity is large, some point may keep being located under the rotor when the rotor restores to be level. If the eccentricity is small, then, it may generate a centrifugal force synchronizing with rotating frequency. Thus, the rotor is caused to vibrate. Rotor imbalance is the most common power machinery fault [2]. 
2.1.2. Rotor Misalignment: The rotors of the machinery and equipments are connected by coupling and form the axis to transmit the motion and torque. Rotor misalignment may be often resulted from installation errors, casing deformation, uneven ground and other factors [3]. Rotor misalignment generally includes axis misalignment and bearing misalignment. Bearing misalignment may often only have an impact on the oil film morphology of the bearing and the distribution of loads and may not result in vibration. Thus, it is generally accepted that the rotor misalignment may refer to axis misalignment.

2.1.3. Rotor Rubbing: Both the rotor misalignment and rotor imbalance are likely to enlarge the rotor vibration amplitude and thus the rotors rub with the fixed contacts mutually, causing abnormal vibration. The rubbing may have great impact on the motion of the rotor system and change the rotor's rotating and even change the motion direction of the rotors [4]. Meanwhile, rubbing may cause the dynamic and static parts contact mutually and enlarge the system rigidity, resulting nonlinear vibration of the system.

2.1.4. Rotor Oil Whirl and Oil Whip: Oil film instability (oil whirl and oil whip) is the self-excited vibration caused by the sliding bearing oil film mechanical characteristics. This is a common fault peculiar to high speed, light load and flexible rotor system. In the event of oil film instability, the axis vibration will be increased rapidly in a short time, causing serious damage to the rotor system [5]. Oil whip is the self-excited vibration of rotating shaft caused by oil film effect of the sliding bearing, oil whirl and oil whip belong to the same phenomenon, when conditions permit, oil whirl will develop into oil whip.

\subsection{Rotor System Fault Diagnosis Methods}

The fault characteristic extraction technologies commonly used in rotor system fault diagnosis include signal time-domain analysis, frequency-domain analysis and timefrequency domain analysis [6]. Time-domain analysis can only roughly detect whether equipments fail, while the frequency-domain analysis is used to find out the fault sources through the analysis of each frequency component and has been widely used in fault diagnosis of mechanical equipments.

2.2.1. Time-domain Diagnosis Method: Time domain waveform is the dynamic caused by constant changing transient value of rotor vibration amplitude with time changing [7]. For rotating machinery vibration signal, the signal amplitude is comparative intuitive feature information of rotating machinery vibration signal. Directly analysis and evaluation on the time course of the vibration signal is the most simple and most direct way to fault diagnosis.

In the signal-based time sequence model analysis, the signal sampling value is deemed a time sequence to establish mathematic models. Then, model parameters are used for fault analysis. Assume $x(t)(t=1,2, \ldots, N)$ is a set of stationary random sample data, then, the following equation can be created:

$x(t)=\sum_{k=1}^{n} \varphi_{k} x(t-k)+e(t)$

In Formula (1), $\varphi_{k}(k=1,2, \ldots, n)$ is an autoregressive parameter; $n$ is an autoregressive order; $e(t)$ is the residual of the model, which is a normal independently distributed random sequence with mean of zero and variance of $\sigma_{a}^{2}$.

2.2.2. Frequency-domain Diagnosis Method: For the mechanical fault diagnosis, the time domain analysis can only provide very limited amount of information, it is commonly used in fault diagnosis for the simple diagnosis of equipment failure. 
Frequency domain analysis is to change the time domain signals with time as abscissa by the Fourier transformation into frequency domain signals with frequency as abscissa, thereby a kind of analysis method is obtained about the amplitude and phase information of the original time domain signal frequency components. Find the source of trouble through the analysis of the frequency components and referring to equipment run time frequency characteristics [8]. Frequency domain analysis method has been widely used in fault diagnosis of mechanical equipment.

The Fourier transformation is an important tool for frequency structure analysis [9]. For a time-domain signal $x(t)$, its Fourier transformation is:

$$
X(f)=\int_{-\infty}^{\infty} x(t) e^{-i 2 \pi f t} \mathrm{~d} t
$$

Fourier transformation is performed as to the time-domain waveform of vibration. After Fourier spectrum analysis, amplitudes at each order harmonic frequency have been clearly extracted out, providing a basis for the discrimination of the fault. So, Fourier transformation is effective for extraction of fault feature of the rotor system.

2.2.3. Time-frequency Analysis Diagnosis Method: Main characteristic of timefrequency diagnosis is the time and frequency localization, through the phase plane constituted by two coordinates of time and frequency axis, frequency content of the overall signal can be obtained in local time domain, and distribution and arrangement for each frequency band of the overall signal can also be obtained in local time [10]. For rotor system, a large number of vibration signals are nonstationary and nonlinear when the failure occurs, therefore, time-frequency diagnosis method has been widely used in the rotor system fault diagnosis.

In time-frequency diagnosis method, the wavelet transform is of isometric feature, with high time resolution in the high frequency and high frequency resolution in the low frequency, the "zoom" feature makes wavelet transformation very suitable for mutation signal processing, but this method does not have adaptability, to a certain extent, this restricts the application of wavelet analysis. Hilbert - Huang transformation [11] is a new method of adaptive time-frequency analysis, fundamentally getting rid of the bondage of the theory of Fourier transform and fully explaining the definition of instantaneous frequency, it can make adaptive time-frequency decomposition according to local timevarying characteristics of the signal, it has very high signal-to-noise ratio, can get high time-frequency resolution, has good time-frequency aggregation, and it is very suitable for non-stationary and nonlinear process.

\section{Rotor System Fault Diagnosis based on Improved LMD and Singular Value Decomposition}

\subsection{Local Mean Decomposition Method and its Disadvantages}

The rotor vibration signals are mostly non-stationary and non-linear dynamic signals. It is very difficult to make accurate analysis of signals in time domains and frequency domains by traditional rotor fault diagnosis methods. LMD a new self-adaptive timefrequency analysis method proposed by JonathanS.Smith in 2005. This method is able to, based on the local characteristic time scale, decompose a complex non-stationary signal into a number of PF components [12], very suitable for processing of non-stationary and non-linear signals.

LMD method is used to achieve the signal decomposition via iteration. During the decomposition process, moving average method is used for smoothing as to the initial local mean function and the local envelope function [13]. Thus, it generates end effect problems and smoothing problems unavoidably. The end effect may cause divergence on 
data ends of the local mean function line and the local envelope function line and finally lead to serious distortion of decomposition results. The moving average method may also directly affect the accuracy and rate of LMD decomposition.

\subsection{The Improved LMD Method}

Based on the disadvantages of LMD method mentioned above, this paper puts forward a method for rotor system fault diagnosis based on the improved LMD and singular value decomposition [14]. In this method, wavelet packet decomposition method is firstly used to decompose the signal into a number of wavelet components. Then, LMD decomposition is performed for each wavelet component. Eventually, the PF components are applied to form the initial feature vector matrix and the singular value of such initial feature vector matrix is extracted as an input to the neural network for identification of the rotor system working status and fault types.

3.2.1. Decomposition of Wavelet Packets: The decomposition of wavelet packets [15] is able to perform multi-layer division of the frequency bands and select the appropriate frequency bands self-adaptively according to the signal features. Three-layer wavelet packet decomposition is as shown in Figure 1, in which, S refers to the original signal; A1 represents the low frequencies after decomposition of one layer; D1 represents the high frequencies after decomposition of one layer; D2 refers to the high frequencies and A2 refers to the low frequencies and so on. The decomposition relationship $\mathrm{S}=\mathrm{A} 3+\mathrm{D} 3+\mathrm{D} 2+\mathrm{D} 1$ can be got. So, in this way, the signal can be decomposed to each frequency band. Some frequency bands can be deleted and some can be kept as necessary.

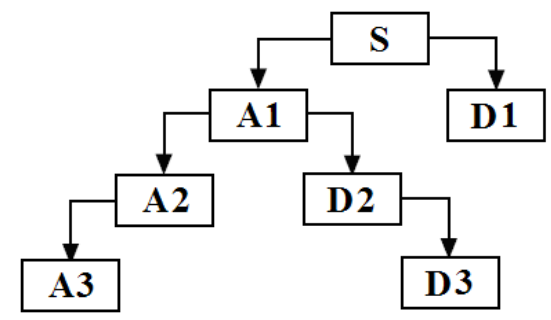

\section{Figure 1. Three-layer Wavelet Packet Decomposition Structure Diagram}

3.2.2. Improvement of LMD: LMD method can deal with the non-stationary signal as the stationary signal, and the inherent nature of signals is retained in the process of decomposition, actually the signal is decomposed into different scales of pure frequency modulated signal and envelope signal. For any signal $\mathrm{x}(\mathrm{t})$, the PF component can be obtained by the method as shown in Literature [12].Wavelet packet decomposition method can map signal to a primary function family formed by flexible wavelet, the amount of information is intact, through the decomposition and reconstruction of different scales, decomposition sequence distributing in different channel can be obtained, not only decomposing the low frequency part, but also decomposing the high frequency part, so as to improve the frequency resolution of the signal pass-band. Before LMD decomposition making wavelet packet decomposition for the signal data can improve the frequency resolution of LMD method [16]. The specific methods for LMD improvement are as follows:

The specific methods for LMD improvement are as follows:

(1) wavelet packet decomposition is performed as to the original signals to gain $n$ wavelet packet components $c_{1}, c_{2}, \ldots, c_{n}$;

(2) LMD decomposition is performed as to the component $c_{1}$ of the wavelet packet to gain $m_{1} \mathrm{PF}$ components $\mathrm{PF}_{11}, \mathrm{PF}_{12}, \ldots \mathrm{PF}_{1 m 1}$ and margin $u_{\mathrm{lm} 1}$; 
(3) add the margin $u_{\mathrm{lm} 1}$ containing a small amount of fault information to the low frequency component $\mathrm{c}_{2}$ to get $c_{2}^{\prime}$;

(4) LMD decomposition is performed as to $c_{2}$ to obtain $m_{2} \mathrm{PF}$ components $\mathrm{PF}_{21}, \mathrm{PF}_{22}$, $\mathrm{PF}_{2 m 2}$ and margin $u_{2 m 2}$;

(5) add the margin $u_{2 \mathrm{~m} 2}$ to the next low frequency component and perform LMD decomposition as to the obtained results. Repeat this again and again until all wavelet components are decomposed and finally, $m_{1}+m_{2}+\ldots+m_{\mathrm{n}}$ PF components are obtained.

\subsection{Singular Value Decomposition}

SVD (Singular Value Decomposition) was first proposed in 1873 by Beltrami [17] to a real matrix, it is a special kind of matrix transformation. Set a matrix $A$ of $N$ rows $M$ columns, and $\operatorname{rank}(A)=r$, to do the following decomposition for it:

$$
A=U S V^{T}=U\left[\begin{array}{rr}
\Sigma & 0 \\
0 & 0
\end{array}\right]^{T}, U * U^{T}=I, V * V^{T}=I
$$

In this formula $\Sigma=\operatorname{diag}\left(\sigma_{1}, \sigma_{2}, \cdots \sigma_{r}\right), \quad \sigma_{1} \geq \sigma_{2} \cdots \geq \sigma_{\mathrm{r}}>0$ is all $r$ non-zero singular value of $A$, is the square root of the $A A^{T}$ or $A^{T} A$ eigenvalue. Corresponding to $\sigma_{i}$ the column vector of $u_{i}$ and $v_{i}$, are respectively called the $i$ singular vectors and the $i$ right singular vectors. For any singular value $\sigma_{i}, u_{i} \sigma_{i} v_{i}^{T}$ is still a matrix of $N$ rows $M$ columns, except for co-dependent rows with equaling to, or just a scale factor difference [18].

Singular value of a matrix is its inherent attribute. It has good stabilities. When small perturbations occur to Matrix $A$, its singular value will change a little. This shows that as a kind of eigenvalue, the singular value is insensitive to the changes caused by the noise, pollution and etc. In addition, the singular value of a matrix also has such characteristics as scale invariance, rotational invariance and dimensionality reduction and compression. Thus, the singular value of a matrix meets the requirements for characteristic extraction in model identification and is able to effectively describe the characteristics of the initial matrix.

\subsection{Improved Rotor System Fault Diagnosis Method}

Figure 2 shows the specific flow chart of the rotor system fault diagnosis method based on the improved LMD and singular value decomposition. First, perform wavelet packet decomposition as to the original signals to gain a number of wavelet components and then perform LMD decomposition as to the obtained wavelet packet components to gain a series of PF components. Each PF component contains different frequency components and different frequencies contain different fault information. Therefore, these PF components can be applied to form the initial feature vector matrix $A$ :

$$
A=\left[\begin{array}{llllll}
P F_{11} & P F_{12} \ldots P F_{1 m_{1}} \ldots P F_{n m_{n}}
\end{array}\right]
$$

In formula (4), $\mathrm{PF}_{n m}$ shows the $m \mathrm{PF}$ of the $n$ wavelet component. So after wavelet packet decomposition and LMD decomposition, the characteristics of the original signal can be represented by the initial eigenvector matrix $A$, make singular value decomposition for the initial matrix $A$. Singular value can effectively describe the characteristics of the initial matrix $A$, namely the failure characteristics of vibration signals, thus the initial matrix singular value can be extract as fault feature vector, inputting neural network to identify working state and fault type for the rotor system. 


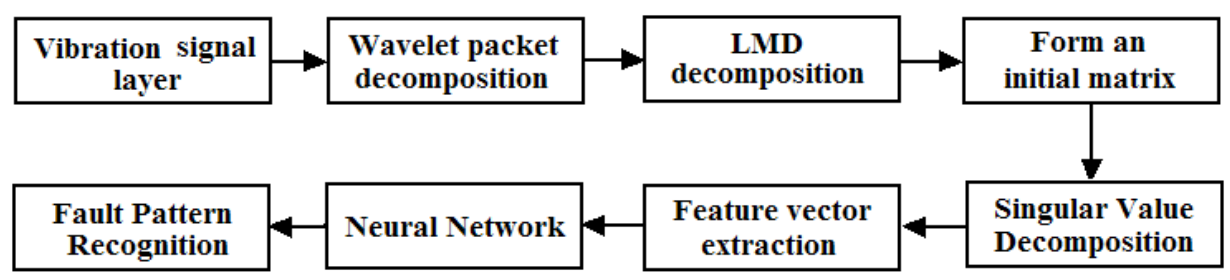

Figure 2. Flow Chart of the Rotor System Fault Diagnosis Method based on the Improved LMD and Singular Value Decomposition

The specific steps for rotor system fault diagnosis are as follows:

(1) according to certain sampling frequency $f_{s}$, four kinds of working state of the rotor system (normal, misalignment, imbalance and oil whirling) are respectively sampled for $\mathrm{N}$ times to get $4 N$ samples;

(2) make wavelet packet decomposition for the sample signal to get several wavelet components;

(3) make further LMD decomposition for each wavelet component to get several PF component to form the initial feature vector matrix A;

(4) make singular value decomposition for the initial feature vector matrix $A$ to get the singular value matrix $\sigma_{j}$ :

$$
\sigma_{j}=\left[\sigma_{j}^{1}, \sigma_{j}^{2}, \ldots, \sigma_{j}^{k}\right]
$$

In this formula $\sigma_{j}^{1} \geq \sigma_{j}^{2} \geq \cdots \geq \sigma_{j}^{k}, j=1,2,3,4$ stand for four different working conditions of normal, misalignment, imbalance and oil whirling;

(5) input the singular value matrix $\sigma_{j}$ as the feature vector into neural network to identify working state and fault type for the rotor.

\section{Experimental Simulation Analysis}

In order to verify the feasibility of this method, 20 sets of vibration signals are detected from the rotor fault test bench in normal, misalignment, imbalance and oil whirl conditions respectively. The sampling frequency is $2048 \mathrm{~Hz}$ and the rotation rate is 6000 rpm. 10 sets of data are extracted randomly from such 4 types of data respectively to be sample data and the remaining data are used as test data.

This paper applies 'dbl0' to perform 2-layer wavelet packet decomposition as to the signals and obtains 4 wavelet components. The first $2 \mathrm{PF}$ components are selected to form the initial feature vector matrix. So, the initial feature vector matrix $A$ consists of $8 \mathrm{PF}$ components. Then, it performs singular value decomposition to such initial feature vector matrix $A$ and works out the singular value matrix $\sigma_{j}^{k}(j=1,2,3,4)$ to be the neural network input. After comparison, the neural network's structure is set as $8 \times 10 \times 4$. In this experiment, the rotor vibration signals are gained in four conditions totally, so, the conditions codes of the output matrix are: normal $(1,0,0,0)$, misalignment $(0,1,0,0)$, imbalance $(0,0,1,0)$, oil whirl $(0,0,0,1)$. For each mode, 10 samples are applied for training. As of the end of the training, the error is 0.0001 and the learning rate is 0.25 . Network training is performed until converge. The well trained neural network is used to classify the test samples. The results show that the neural network is able to identify all fault samples successfully. Table 1 shows the eight test sample characteristic vector and the corresponding network output value is presented in Table 2. 
Table 1. Test Sample Feature Vector

\begin{tabular}{c|c|c|c|c|c|c|c|c}
\hline Status & $\sigma^{1}$ & $\sigma^{2}$ & $\sigma^{3}$ & $\sigma^{4}$ & $\sigma^{5}$ & $\sigma^{6}$ & $\sigma^{7}$ & $\sigma^{8}$ \\
\hline Normal & 164.16 & 95.852 & 80.117 & 32.303 & 20.532 & 3.0664 & 1.8711 & 0.9623 \\
\hline Normal & 157.86 & 87.002 & 74.479 & 42.207 & 20.636 & 6.9351 & 3.6367 & 2.1217 \\
\hline Misalignment & 213.41 & 130.20 & 108.57 & 73.952 & 18.313 & 2.0496 & 1.3544 & 0.9193 \\
\hline Misalignment & 208.93 & 88.117 & 79.516 & 51.261 & 18.538 & 6.0475 & 2.182 & 1.5913 \\
\hline Imbalance & 325.49 & 92.247 & 48.611 & 35.484 & 21.207 & 4.2132 & 2.1998 & 1.0997 \\
\hline Imbalance & 325.16 & 99.394 & 58.033 & 47.259 & 33.468 & 28.791 & 23.056 & 14.477 \\
\hline Oil whirl & 151.56 & 74.648 & 32.557 & 22.604 & 16.481 & 4.0626 & 2.1891 & 1.2423 \\
\hline Oil whirl & 153.98 & 67.556 & 31.151 & 17.935 & 16.117 & 1.3638 & 1.1085 & 0.7467 \\
\hline
\end{tabular}

Table 2. The Diagnosis Output Result

\begin{tabular}{|c|c|c|c|c|c|}
\hline Status & \multicolumn{4}{|c|}{ Network Output } & $\begin{array}{c}\text { Results } \\
\text { Identification }\end{array}$ \\
\hline Normal & 1.0318 & -0.0067 & -0.0006 & -0.0237 & \multirow{2}{*}{ right } \\
\hline Normal & 1.0365 & -0.0114 & -0.0235 & -0.0009 & \\
\hline Misalignment & -0.0341 & 0.8726 & 0.0413 & 0.1218 & \multirow{2}{*}{ right } \\
\hline Misalignment & -0.0119 & 1.0376 & -0.0407 & 0.0168 & \\
\hline Imbalance & 0.0643 & -0.0199 & 0.9033 & -0.0476 & \multirow{2}{*}{ right } \\
\hline Imbalance & -0.0655 & 0.0151 & 1.0345 & 0.0159 & \\
\hline Oil whirl & -0.0465 & -0.0041 & 0.0040 & 1.0464 & \multirow{2}{*}{ right } \\
\hline Oil whirl & 0.0634 & -0.0071 & 0.0028 & 0.8411 & \\
\hline
\end{tabular}

\section{Conclusions}

This paper uses a wavelet-LMD method to decompose the rotor signals to form an initial feature vector matrix and then performs singular value decomposition as to the vector matrix to work out the singular value matrix which is able to represent the rotor fault information characteristics and used the eigenvector to input to the neural network. Experimental analysis results show that the method of combination of wavelet-LMD and singular value decomposition can be effectively used in the rotor system fault diagnosis. In the future, it is required to further improve the function of the fault diagnosis software.

\section{References}

[1] G. Poetsch, "Pantograph/catenary dynamics and control", Vehicle System Dynamics, vol. 30, (1997), pp. 159-195.

[2] L. Cohen, "Time-frequency distribution a review", Proceedings of the IEEE, vol. 77, no. 7, (1989), pp. 941-981.

[3] J. S. Smith, "The local mean decomposition and its application to EEG perception data", Journal of the Royal Society Interface, vol. 2, no. 5, (2005),pp. 443-454.

[4] W. Sweldens, "The lifting scheme: A Construction of Second Generation Wavelets", SIAM Journal of Mathematical Analysis, vol. 29, no. 2, (1998), pp. 511-546.

[5] S. R. Qin and Y. M. Zhong, "A new algorithm of Hilbert-Huang transform", Mechanical Systems and Signal Processing, vol. 20, no. 8, (2006), pp. 1941-1952.

[6] P. D. MeFadden, "Model for the vibration Produeed by a single Point defect in a rolling element bearing", Journal of Sound and Vibration, vol. 96, (1984), pp. 69-82.

[7] Y. Kim, B. Lim and W. S. Cheoung, "Fault detection in a ball bearing system using a moving window", Mechanical Systems and Signal Processing, vol. 5, no. 6, (1991), pp. 461-473. 
[8] D. Brie, "Modelling of the spalled rolling element bearing vibration signal: an overview and some new results", Mechanical Systems and Signal Processing, vol. 14, no. 3, (2000), pp. 353-369.

[9] W. J. Williams and E. J. Zalubas, "Helicopter transmission fault detection via time-frequency, scale and spectral methods", Mechanical System and Signal Processing, vol. 14, no. 4, (2000), pp. 545-559.

[10] R. B. RandaII, J. Antoni and S. Chobsaard, "The relationship between spectral correlation and envelope analysis in the diagnostics of bearing faults and other cyclostationary machine Signals", Mechanical Systems and Signal Processing, vol. 15, no. 5, (2001), pp. 945-962.

[11] J. Courrech, "Envelope analysis for effective rolling element fault detection-fact or fiction", UPTIME Magzine, vol. 8, no. 1, (2000).

[12] Y. F. Wang and P. J. Kootsookos, "Modeling of low shaft speed bearing faults for condition monitoring”, Mechanical Systems and Signal Processing, vol. 12, no. 3, (1998), pp. 415-426.

[13] D. Ho and R. B. Randall, "Optimisation of bearing diagnostic techniques using simulated and actual bearing fault signals", Mechanical Systems and Signal Processing, vol. 14, no. 5, (2000), pp. 763-788.

[14] N. E. Huang, M.-L. C. Wu and S. R. Long, "A confidence limit for the empirical mode decomposition and Hilbert spectral analysis", Proc. R. Soc. Lond. A, vol. 459, (2003), pp. 2317-2345.

[15] D. Yongjun and W. Wei, "Boundary processing technique in EMD method and Hilbert transform", Chinese Science Bulletin, vol. 46, no. 11, (2001), pp. 257-263.

[16] C. Junsheng, Y. Dejie and Y. Yu, "Research on the intrinsic mode function (IMF) criterion in EMD method", Mechanical Systems and Signal Processing, vol. 20, no. 4, (2006), pp. 817-824.

[17] L. Bottou, C. Cortes and J. S. Denker, "Comparison of classifier methods: a case study in handwritten digit recognition", Presented at Proceedings of the 12th IAPR International, vol. 12, (1994), pp. 77-82.

[18] S. Knerr, L. Personnaz and G. Dreyfus, "Single-layer learning revisited: A stepwise procedure for building and training a neural network", Neurocomputing: Algorithms, Architectures and Applications, J. Fogelman, Ed. New York: Springer-Verlag, (1990).

\section{Author}

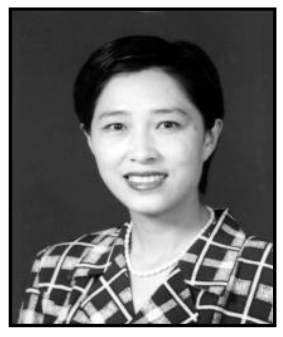

Hong Zhang (1968--), she is a professor, she graduated from Department of Electronic Engineering of Shandong University in 1991, she was majored in electronic information, obtaining a bachelor's degree in engineering, in 2006 she graduated from College of information and control engineering of Shandong University of Technology, obtaining master's degree in engineering. Her main research directions include intelligent control, network and communication. (E-mail: zhanghongwf@126.com) 